\title{
Live-Cell Imaging of Rhabdovirus-Induced Morphological Changes in Plant Nuclear Membranes
}

\author{
Michael Goodin, ${ }^{1}$ Sharon Yelton, ${ }^{1}$ Debasish Ghosh, ${ }^{2}$ Stephanie Mathews, ${ }^{1}$ and Judith Lesnaw ${ }^{2}$ \\ ${ }^{1}$ Department of Plant Pathology and ${ }^{2}$ Department of Biology, University of Kentucky, Lexington 40546, U.S.A.
}

Submitted 5 August 2004. Accepted 9 March 2005.

\begin{abstract}
Potato yellow dwarf virus (PYDV) and Sonchus yellow net virus (SYNV) belong to the genus Nucleorhabdovirus. These viruses replicate in nuclei of infected cells and mature virions accumulate in the perinuclear space after budding through the inner nuclear membrane. Infection of transgenic Nicotiana benthamiana 16c plants (which constitutively express green fluorescent protein (GFP) targeted to endomembranes) with PYDV or SYNV resulted in virusspecific patterns of accumulation of both GFP and membranes within nuclei. Using immunolocalization and a lipophilic fluorescent dye, we show that the sites of the relocalized membranes were coincident with foci of accumulation of the SYNV nucleocapsid protein. In contrast to the effects of PYDV and SYNV, inoculation of $16 \mathrm{c}$ plants with plusstrand RNA viruses did not result in accumulation of intranuclear GFP. Instead, such infections resulted in accumulation of GFP around nuclei, in a manner consistent with proliferation of the endoplasmic reticulum. We propose that the relocalization of GFP in $16 \mathrm{c}$ plants can be used to study sites of rhabdovirus accumulation in live cells. This study is the first to use live-cell imaging to characterize the effects of rhabdoviruses on plant nuclear membranes.
\end{abstract}

Additional keywords: glycoprotein, nucleus, viroplasm.

How plant rhabdoviruses affect the cell biology of their host cells is poorly understood. Although there is a considerable body of literature relating changes in host cell endomembranes in response to infection by plus-strand RNA viruses, only limited attention has been given to the phytorhabdoviruses. In this report, we present data from experiments conducted with two rhabdoviruses that induce accumulation of GFP in nuclei of cells in infected Nicotiana benthamiana 16c plants, which constitutively express green fluorescent protein (GFP) targeted to endomembranes (Brigneti et al. 1998; Reichel and Beachy 1998; Ruiz et al. 1998; Turner et al. 2004).

The aphid- and mechanically transmissible Sonchus yellow net virus (SYNV) is the most extensively characterized of all the plant rhabdoviruses at the molecular, biochemical, and cell biology levels (Goodin et al. 2001, 2002; Jackson et al. 1987; Martins et al. 1998). The bacilliform particles of SYNV are composed of infectious nucleocapsids surrounded by a phospholipid membrane that can be purified by density gradient centrifugation of nonionic detergent-treated virions (Wagner and Jackson 1997). Nucleocapsids are ribonucleoprotein (RNP) complexes that consist of the 13,720-nucleotide (nt) negativestrand genomic RNA (Wagner et al. 1996; Wagner and Jackson

Corresponding author: Michael Goodin, Ph.D.; Telephone: 859-257-7445 ext. 80725; Fax: 859-323-1961; E-mail: mgoodin@uky.edu
1997) encapsidated by three associated proteins, namely the nucleocapsid $(\mathrm{N})$, phosphoprotein $(\mathrm{P})$, and polymerase $(\mathrm{L})$ proteins. The membrane fraction contains a glycoprotein $(\mathrm{G})$ that protrudes from the surface of the virion (Goldberg et al. 1991) and an additional protein designated sc4 (Scholthof et al. 1994) that may play role in virus cell-to-cell movement (Melcher 2000). The matrix (M) protein (Hillman et al. 1990) is thought to associate with the $\mathrm{G}$ protein, presumably aiding in condensation of the nucleocapsids during virion maturation. Electron microscopy studies suggest that, during morphogenesis, the condensed nucleocapsids acquire the $G$ protein and a host-derived lipid envelope as they bud through the inner nuclear membrane and accumulate as mature particles in the perinuclear space (van Beek et al. 1985).

In contrast to SYNV, the molecular and cellular biology of Potato yellow dwarf virus (PYDV), the type member of the genus Nucleorhabdovirus, has not been extensively explored (Hsu and Black 1973, 1974; MacLeod et al. 1966). However, PYDV is an attractive model for rhabdovirus research because it is also mechanically transmissible and, as we show below, affects plant cell membranes in a manner in stark contrast to the effects of SYNV.

Common to infection of plant cells by both SYNV and PYDV is the formation of large inclusions or "viroplasma" in nuclei of infected cells (MacLeod et al. 1966; Martins et al 1998). Characterization of viroplasma by light and electron microscopy suggests that they are sites of viral replication because they contain the $\mathrm{N}, \mathrm{P}$, and L proteins and the genomic and antigenomic RNAs (Christie and Edwardson 1977; Martins et al. 1998).

In order to circumvent both the need to work with fixed tissues and the use of engineered viruses, we explored the potential of $16 \mathrm{c}$ plants to monitor sites of rhabdovirus accumulation. Northern hybridization experiments failed to show a downregulation of GFP gene expression in SYNV-infected plants (data not shown), as might be predicted if plant rhabdoviruses retained the ability to shut off host gene expression as is observed in the case of rhabdoviruses with animal hosts (Petersen et al. 2000, 2002). Instead, fluorescence microscopy experiments, conducted in live cells, revealed that GFP accumulated in loci within and around nuclei, thus providing a means to mark rhabdovirus infections sites. We also found that, even though both SYNV and PYDV induced nuclear accumulation of GFP, the pattern of localization was unique to each virus. In contrast to our observations with rhabdoviruses, we determined that infection of $16 \mathrm{c}$ plants with representative viruses from the Potyvirus, Tospovirus, and Tobravirus genera did not result in the accumulation of GFP within nuclei.

Taken together, our experiments with SYNV and PYDV extend related studies, conducted with plus-strand RNA viruses, by providing novel insight into the nuclear membrane dynamics 
associated with virus infection. Additionally, our findings provide a facile means to mark sites of rhabdovirus accumulation in live-plant cells.

\section{RESULTS}

GFP accumulation in nuclei of rhabdovirus-infected leaves. GFP in mock-inoculated 16c N. benthamiana plants was found to be associated primarily with membranes around the periphery of cells (Fig. 1). In contrast, GFP accumulated in nuclei of SYNV- or PYDV-infected tissue. Laser-scanning confocal microscopy (LSCM) and counter-staining with the DNAselective dye 4'-6-diamidino-2-phenylindole dihydrochloride (DAPI) confirmed that GFP accumulated within nuclei in SYNV-infected tissues. (Fig. 1). In SYNV-infected tissues, GFP accumulated mostly within the DAPI-stainable portion of the nucleus (Fig. 1B through D) in contrast to PYDV-infected cells, where GFP accumulated on the periphery of the nucleus as well as in a region that could not be stained with DAPI (Fig. 1F through $\mathrm{H}$ ).

We found that GFP accumulated at multiple foci within nuclei of SYNV-infected tissue, which was consistent with reports of the location of viroplasm formation (Martins et al. 1998) (Fig. 1C). In contrast, accumulation of PYDV-induced viroplasm was found to be in internal and external nuclear loci (MacLeod et al. 1966). The optical section in Figure 1G showed that GFP accumulation was consistent with these findings. However, to make a more definitive assignment, we produced three-dimensional (3-D) projections derived from optical sections taken through the GFP accumulated in nuclei of cells in PYDV-infected tissues (Fig. 2). The 3-D images were rotated so that multiple projections could be made (Fig. 2A through C). Projections suggested that PYDV-induced loci of GFP accumulation were both within and outside of the nucleus (Fig. 2B, white arrow). To make definitive assignments, optical sections were made through the $\mathrm{X}, \mathrm{Y}$, and $\mathrm{Z}$ planes of the pro-

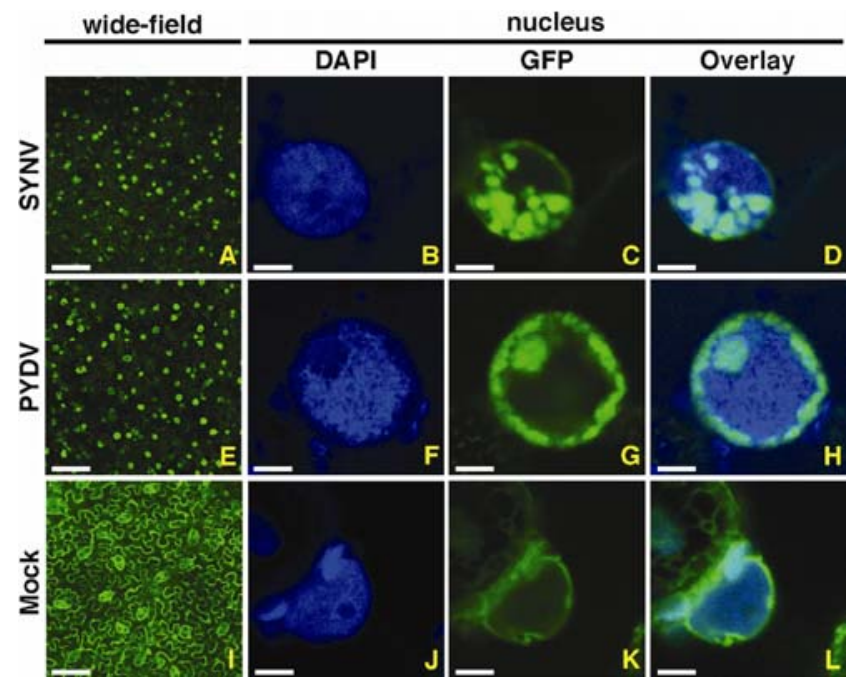

Fig. 1. Confocal micrographs of leaf epidermal cells of Nicotiana benthamiana 16c plants inoculated with A through D, Sonchus yellow net virus (SYNV), E through $\mathbf{H}$, Potato yellow dwarf virus (PYDV), or $\mathbf{I}$ through $\mathbf{L}$, mock inoculated. A, E, and I, Detection of green fluorescent protein (GFP) fluorescence in wide-field micrographs of epidermal cells of SYNV-, PYDV-, and mock-inoculated leaves, respectively. Scale-bar = $200 \mu \mathrm{m}$. B through D, F through $\mathbf{H}$, and $\mathbf{J}$ through $\mathbf{L}$, Detection of 4'-6diamidino-2-phenylindole dihydrochloride (DAPI) and GFP fluorescence, and the overlay of these images, in single nuclei of leaf epidermal cells of SYNV-, PYDV- and mock-inoculated leaves, respectively. Scale bar $=5$ $\mu \mathrm{m}$. Exposure times for micrographs were adjusted to provide correct acquisition of fluorescence signal from the brightest areas within cells. jection shown in Figure 2C. The presence of a large area of GFP fluorescence (Fig. 2D, white arrow) indicated that a large area of accumulated GFP was located within this nucleus. Additional sections showed that most of this fluorescence was external to the area of DAPI fluorescence (Fig. 2E and F).

\section{Accumulation of GFP}

within nuclei in multiple types of tissues.

In systemically infected leaves of $N$. benthamiana plants inoculated with SYNV or PYDV, nuclear accumulation of GFP was observed first in the primary veins, followed by secondary veins and, ultimately, at late stages of infection, in epidermal cells adjacent to tertiary and quaternary veins (data not shown). Therefore, based on the hypothesis that GFP accumulated in nuclei of virus-infected cells per se, we considered that a gradient in the degree of accumulated GFP should occur from the point source of the primary vein to cells that were devoid of intranuclear GFP. We observed that this gradient occurred over a distance of approximately three to four leaf epidermal cells in leaves infected with PDYV (Fig. 3A and B). Similar gradients of GFP accumulation were observed in SYNV-infected tissues (data not shown).

It is not currently possible to produce recombinant plant rhabdoviruses engineered to express GFP; therefore, we tested the possibility that accumulation of GFP in nuclei correlated with systemic spread of these viruses. In the cases of both SYNV and PYDV, GFP accumulated in nuclei of leaf cells (Fig. 3A and B), roots (Fig. 3F through $\mathrm{H}$ ), or trichomes (Fig. 3I through $\mathrm{K}$ ). Accumulation of GFP was not observed in nuclei of root (Fig. $3 \mathrm{~F}$ through $\mathrm{H}$ ) or shoot meristematic cells (data not shown). However, we cannot rule out that SYNV did invade these tissues because we observed that GFP expression in meristematic tissues of 16c plants appeared to be lower compared with levels in other cell types, particularly leaf epidermal cells.
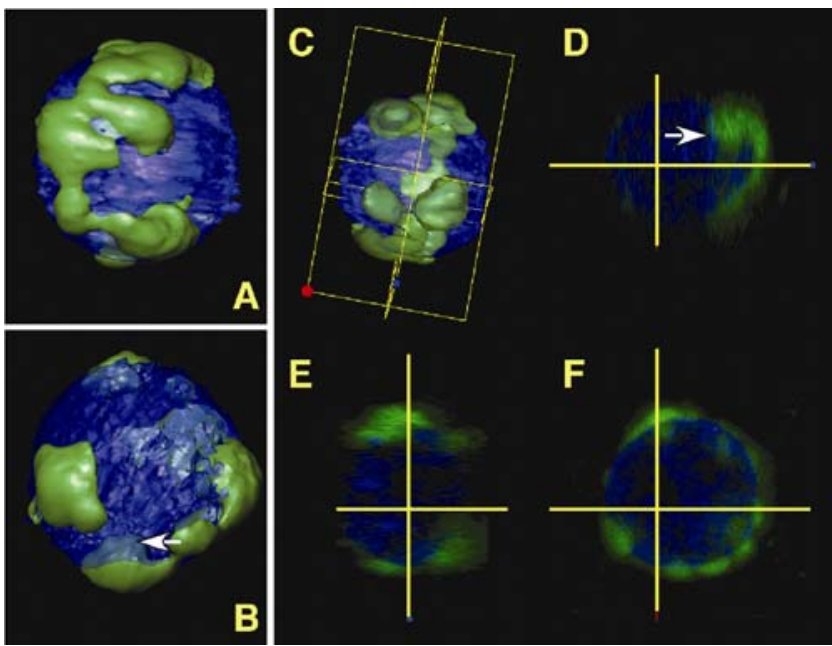

Fig. 2. Three-dimensional (3-D) reconstruction of confocal optical sections taken through a nucleus of a leaf epidermal cell in Potato yellow dwarf virus-infected tissue. A through $\mathbf{C}$, Projections taken after rotation of 3-D model; 4'-6-diamidino-2-phenylindole dihydrochloride (DAPI) fluorescence is shown in blue and that of green fluorescent protein (GFP) in green. The transparency of DAPI in the reconstruction was adjusted to $50 \%$ so that internal GFP-marked structures could be seen (gray). A, Projection in which GFP accumulation is shown primarily on the outer surface of the nucleus. B, Projection in which accumulated GFP appeared to protrude from inside of the nucleus to the outside (white arrow). $\mathbf{C}$, Projection through which optical sections were made in the $\mathrm{x}_{-}^{-}, \mathrm{y}_{-}$, and $\mathrm{z}-$ planes to produce images D to F. D, Section through the nucleus showing that GFP accumulated within and outside of the area of DAPI fluorescence. $\mathbf{E}$ through $\mathbf{F}$, Optical sections showing that the majority of GFP fluorescence was detected outside of the area of DAPI fluorescence. 
GFP in virus-infected 16c plants colocalizes with the SYNV-N protein.

The N protein of SYNV accumulates exclusively in the nucleus where it associates with genomic-length viral RNAs in viroplasma and mature virions (Goodin et al. 2001, 2002; Martins et al. 1998; Wagner et al. 1996; Wagner and Jackson 1997). Therefore, using anti-N and anti-GFP polyclonal antibodies, we conducted co-immunolocalization experiments to determine whether the foci of relocalized GFP in infected 16c plants were coincident with those for SYNV-N accumulation (Fig. 4). These experiments clearly revealed that regions within nuclei that reacted most intensely with $\mathrm{N}$ antibodies were in the same location as sites of accumulation of GFP (Fig. 4F through $\mathrm{J}$ ). In contrast, no fluorescence signal, above
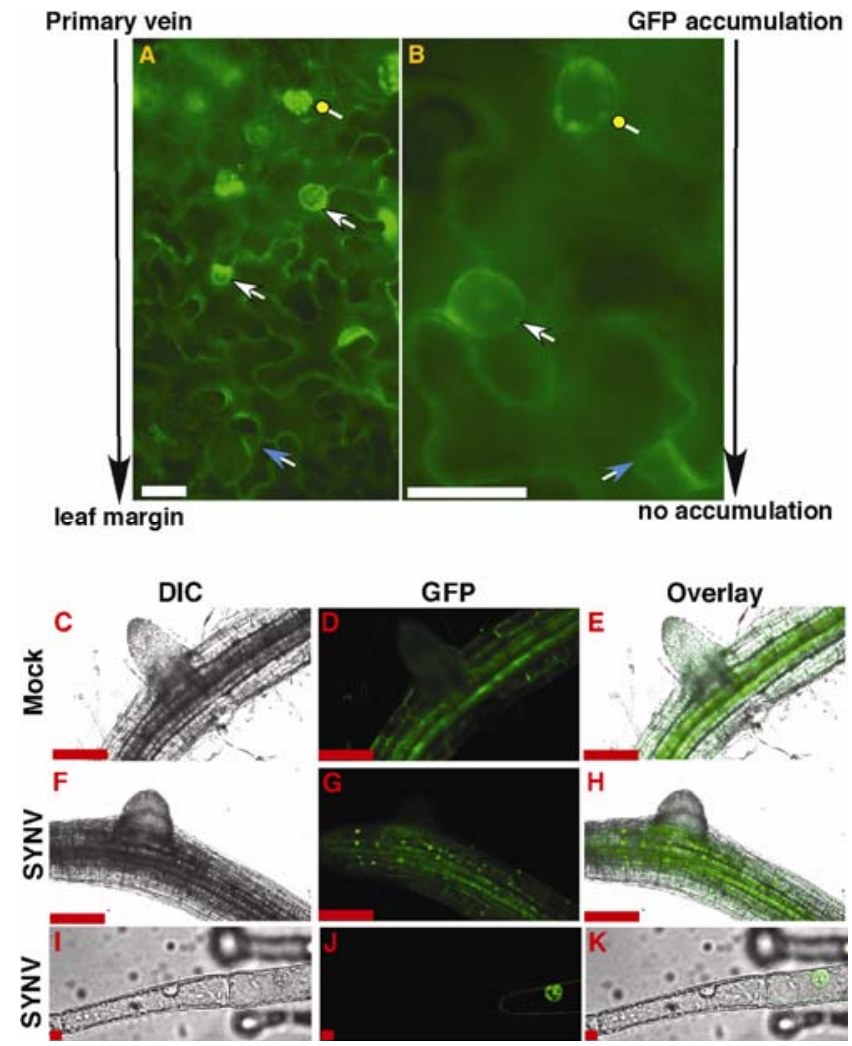

Fig. 3. Epifluorescence micrographs of green fluorescent protein (GFP) accumulation in nuclei of different types of cells. A, Wide-field micrograph of GFP accumulation in epidermal cells of a Potato yellow dwarf virus-infected leaf. Note that GFP in nuclei in the upper portion of the micrograph (yellow marker) accumulated in many foci. Cells in an intermediate region (white arrows) have fewer foci of accumulation, and nuclei in cells at the bottom of the micrograph are devoid of accumulated GFP. Scale bar $=10 \mu \mathrm{m}$. B, High-magnification micrograph of three contiguous cells at the infection front. Note that accumulation of GFP in the middle nucleus (white arrow) has few foci of GFP accumulation, whereas nuclei in cells above (yellow marker) have greater accumulation and that below (blue marker) is devoid of intranuclear GFP. Scale bar $=20$ $\mu \mathrm{m}$. $\mathbf{C}$ through $\mathbf{K}$, Accumulation of GFP in nuclei of root and trichome tissues. $\mathbf{C}$ through $\mathbf{H}$, Differential interference contrast (DIC), GFP fluorescence micrographs, and the merged images (overlay) of roots of Nicotiana benthamiana $16 \mathrm{c}$ plants. C, DIC image of roots of mock-inoculated plants. D, GFP fluorescence of root shown in C. E, Merged image of those shown in C and D. F, DIC image of roots of plants inoculated with Sonchus yellow net virus (SYNV). G, GFP fluorescence of root shown in C. Note punctate foci of GFP fluorescence in the mature tissue and lack of any fluorescence in the root primordium. $\mathbf{H}$, Merged image of those shown in F and G. I, DIC image of the basal (right) and middle cells (left) of a single trichome on a SYNV-infected leaf. J, GFP fluorescence in the nucleus of the basal cell but not the middle cell. $\mathbf{K}$, Overlay of images shown in I and J. Scale bar $=200$ and $10 \mu \mathrm{m}$ for $\mathrm{C}$ through $\mathrm{H}$ and $\mathrm{I}$ through $\mathrm{K}$, respectively. background, was detected by the $\mathrm{N}$ antibodies in mock-inoculated tissues (Fig. 4A through E).

It also should be noted that immunodetection of GFP was necessary for these experiments because the harsh fixation process used to prepare the leaf samples included the use of paraformaldehyde and extended periods of heating at $60^{\circ} \mathrm{C}$. As a result, no detection of direct fluorescence of GFP could be observed in fixed tissues (data not shown).

\section{GFP expressed in 16c plants is membrane-associated and these membranes are internalized within nuclei upon infection by rhabdoviruses.}

Having established that infection by rhabdoviruses alters the localization of GFP in 16c plants (Fig. 1) and that, at least for SYNV, this area of relocalization was coincident with sites of virus accumulation (Fig. 4), we then sought to resolve the nature of the environment in which the relocalized GFP accumulated. To make this determination, we employed a lipophilic dye that specifically stains endomembranes, including the endoplasmic reticulum (ER) and nuclear membranes (Fig. 5). Live-cell imaging revealed that GFP expressed in 16c plants was membrane associated (Fig. 5A through E). We also were able to demonstrate for both SYNV and PYDV that the virusrelocalized GFP remains associated with membranes (Fig. 5F through P).

In order to test the hypothesis that the observed localization of GFP within nuclei was specific to nucleorhabdoviruses and not a product of virus infection per se, we inoculated $16 \mathrm{c}$ plants with a variety of viruses that replicate in the cytoplasm of infected cells. The viruses chosen for these experiments were Tobacco etch virus (TEV; genus Potyvirus), Impatiens necrotic spot virus (INSV; genus Tospovirus), and Tobacco rattle virus (TRV; genus Tobravirus). TEV was chosen because infection of this virus is known to result in the formation of large proteinaceous inclusions in the cytoplasm as well as in the nucleus (Baunoch et al. 1991; Li et al. 1997; Schaad et al. 1996). INSV was chosen in lieu of an available cytorhabdovirus because it is an enveloped virus that replicates in the cytoplasm. TRV was chosen because it is known to infect meristematic tissues, which were sites devoid of GFP accumulation in nuclei of rhabdovirus-infected cells.

Neither TEV, INSV (data not shown), nor TRV (data not shown) induced the formation of discrete foci of GFP inclusions. Instead, we observed diffuse GFP accumulation adjacent

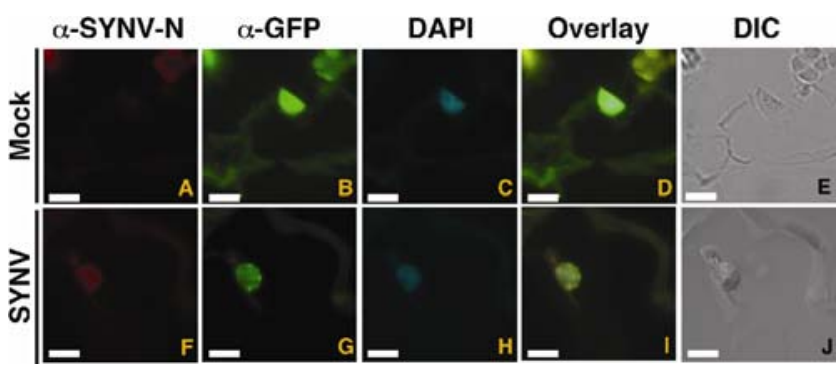

Fig. 4. Colocalization of the Sonchus yellow net virus (SYNV)-N and green fluorescent protein (GFP) proteins. A through E, Micrographs of a cell in fixed mock-inoculated tissue of Nicotiana benthamiana $16 \mathrm{c}$ plants. The fluorescence in the upper right corner of panels A, B, and D was derived from chlorophyll autofluorescence. A, No detection of SYNV-N in mock-inoculated tissue. B, Detection of GFP. C, 4'-6-Diamidino-2-phenylindole dihydrochloride (DAPI) fluorescence. D, An overlay of micrographs A through C. E, Differential interference contrast (DIC) image of cell observed in A through $\mathbf{C}$. $\mathbf{F}$ through $\mathbf{J}$, Micrographs of a cell in fixed SYNV-infected tissue of $16 \mathrm{c}$ plants. F. $\alpha-S Y N V-N$ was used to detect the presence of the N protein. G, Detection of GFP. H, DAPI fluorescence I, An overlay of micrographs F through H. J, DIC image of cell observed in F through H. Scale bar $=20 \mu \mathrm{m}$. 
to, but not within, the nuclei (Fig. 5Q through S). The site of this accumulation may be ER, but no further experiments were conducted to confirm this assumption. Except for this amorphous accumulation of GFP around nuclei, no significant change in the size or shape of the nuclei was observed compared with those of mock-inoculated 16c plants (Fig. 5Q through $\mathrm{S}$; data not shown). Interestingly, co-inoculation of 16c plants with SYNV and TEV resulted in the formation of both nuclear accumulation of GFP and proliferation of membranes surrounding the nuclei (Fig. 5T).

\section{DISCUSSION}

We have used 16c plants that express GFP containing a carboxy-terminal ER-retention signal to study nuclear membrane dynamics in living rhabdovirus-infected cells. As demonstrated in previous studies, GFP targeted to the ER is also transported to the contiguous outer nuclear envelope and, in turn, to the inner nuclear envelope (INE) (Ellenberg and LippincottSchwartz 1999; Ellenberg et al. 1997). Moreover, GFP containing a carboxy-terminal KDEL ER retention signal has been used to study nuclear membrane morphology and dynamics in onion and tobacco cells (Collings et al. 2000). Consistent with

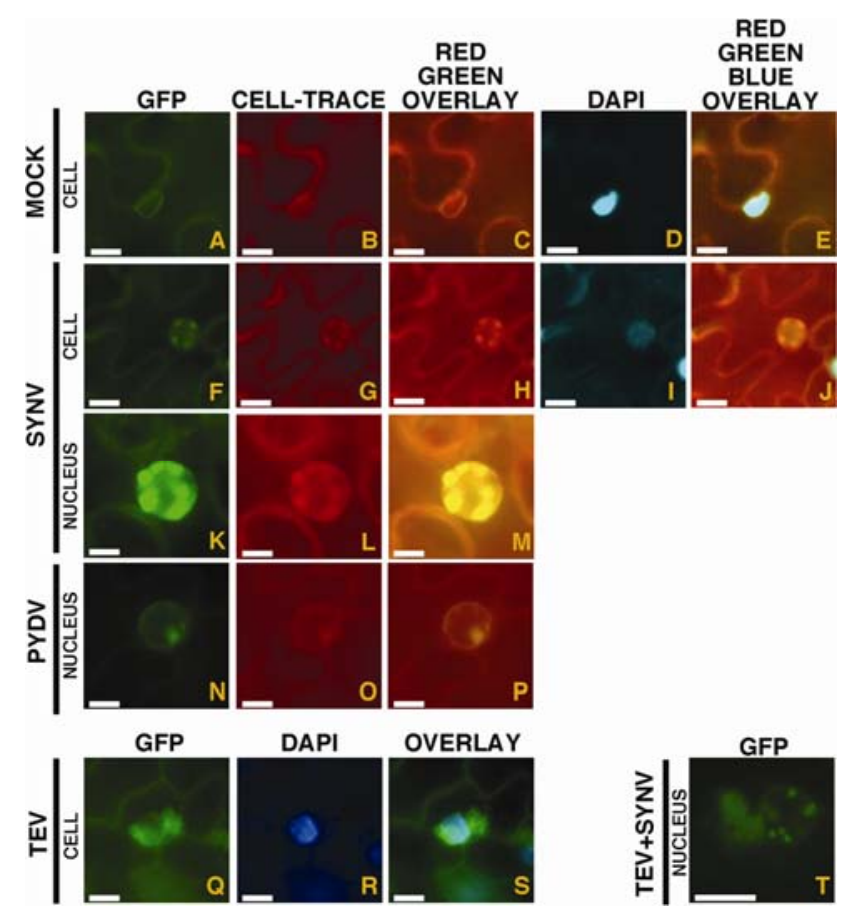

Fig. 5. Epifluorescence micrographs of green fluorescent protein (GFP) fluorescence in both mock- and virus-infected Nicotiana benthamiana 16c plants counterstained with lipophilic- (CELL-TRACE) and DNA-selective (4'-6-diamidino-2-phenylindole dihydrochloride [DAPI]) dyes. A through $\mathbf{E}$, Micrographs of a cell in mock-inoculated leaf tissue. A, GFP fluorescence. B, Fluorescence from CELL-TRACE. C, Overlay of images A and B. D, Fluorescence from DAPI. E, An overlay of images A, B, and D establishes the position of the nuclear envelope in images A and B. F through J, Micrographs of a cell in Sonchus yellow net virus (SYNV)inoculated leaf tissue. F, GFP fluorescence. G, Fluorescence from CELLTRACE. H, Overlay of F and G. I, Fluorescence from DAPI. J, Overlay of F, G, and I. K, High magnification of GFP fluorescence from an SYNVinfected nucleus. $\mathbf{L}$, Fluorescence from CELL-TRACE in the same nucleus shown in K. M, Overlay of K and L. N, High-magnification of GFP fluorescence from a Potato yellow dwarf virus (PYDV)-infected nucleus. O, Fluorescence from CELL-TRACE the same nucleus shown in N. P, Overlay of $\mathbf{N}$ and O. $\mathbf{Q}$ through $\mathbf{S}$, Micrographs and overlays of a single Tobacco etch virus (TEV)-infected cell. Q, GFP fluorescence. R, DAPI-stained nucleus. S, Overlay of Q and R. T, GFP fluorescence from a nucleus of a cell in tissue co-inoculated with TEV and SYNV. these reports, we have shown that GFP expressed in 16c plants colocalizes with an endomembrane-specific dye that stains both ER and nuclear membranes (Fig. 5). Therefore, 16c plants are uniquely suited for cell biology studies with SYNV and PYDV, which are believed to undergo morphogenesis on the INE (MacLeod et al. 1966; van Beek et al. 1985). Studies similar to ours have been conducted with ER- or INE-targeted GFP to study membrane diffusion rates (Dayel et al. 1999), dynamics of nuclear membranes, or the effect of virus infections on ER membranes (Carette et al. 2000; Reichel and Beachy, 1998; Turner et al. 2004). Our data extend these studies by providing novel insight into the cell biology of nucleorhabdoviruses, which have markedly different effects on plant nuclear membranes when compared with plus-strand RNA viruses.

To date, previous studies have employed light and electron microscopy for the characterization of rhabdovirus-induced viroplasma. These studies suggested that establishment of the viroplasm results in the swelling of infected nuclei as well as accumulation of mature virions in the perinuclear space of infected nuclei (Ismail et al. 1987; Jackson et al. 1987; MacLeod et al. 1966; Martins et al. 1998). We have shown, using fluorescence microscopy and co-immunolocalization with the SYNV-N protein, that GFP accumulates in SYNVand PYDV-infected cells in loci consistent with sites where viroplasma are known to accumulate within nuclei of virusinfected tissues (Figs. 1 and 4). The precise colocalization of SYNV-N and GFP excluded the possibility of nonspecific aggregation of GFP protein in virus-infected cells (Fig. 4). Moreover, the finding that GFP fluorescence was always coincident with that of a lipophilic dye confirmed that both GFP-KDEL and nuclear membranes accumulated at the same sites as SYNV (Fig. 5). No evidence for the formation of GFP aggregates that did not colocalize with membranes was found. Although we have yet to determine whether GFP is incorporated into virions, as these data might suggest, it is clear from these studies that the observed alterations in the patterns of GFP localization in $16 \mathrm{c}$ plants was due to effects on plant cell membranes and not GFP protein per se. Further support for this conclusion was provided by experiments conducted with plants infected with TEV. Although aggregates of the NIa and NIb proteins of TEV accumulate within nuclei, no intranuclear accumulation of GFP was observed in cells of TEV-infected tissue (Fig. 5). This suggested that accumulation of viral protein aggregates in nuclei was not the sole determinant for GFP relocalization. In contrast to the localization patterns observed in rhabdovirus-infected tissues, GFP accumulated around the periphery of nuclei in TEV-infected cells. Such a locale was consistent with proliferation of ER, as has been observed in transgenic plants that express ER-targeted GFP infected with Red clover necrotic mosaic virus (RCNMV) (Turner et al. 2004), Cowpea mosaic virus (CPMV) (Carette et al. 2000), and Tobacco mosaic virus (TMV) (Reichel and Beachy 1998).

In contrast to the membrane proliferation observed with TEV, RCNMV, CPMV, and TMV, the replication factor 1a of Brome mosaic virus localizes to outer perinuclear ER membranes, where it induces formation of approximately $60-\mathrm{nm}$ vesicular invaginations, or "spherules", that function as compartments or miniorganelles for RNA replication (RestrepoHartwig and Ahlquist 1996, 1999; Schwartz et al. 2002, 2004). The formation of such spherules has led to the description of parallels in form and function of plus-strand RNA virus replication complexes and retrovirus capsids (Schwartz et al. 2002, 2004). Our results with SYNV, which induces formation of membrane-bound spherules within infected nuclei, suggests that this comparison may be extended to include at least some members of the family Mononegavirales. Nevertheless, it is 
unlikely that rhabdovirus polymerase proteins could be responsible for observed membrane relocalization because these proteins typically are associated only with the viral nucleocapsids and are not membrane-associated (Hammond et al. 1992a and b; Qanungo et al. 2004). It is probable that the SYNV G protein is responsible for the alteration of host membranes because overexpression of $\mathrm{G}$ proteins encoded by rhabdoviruses with animal hosts results in alteration of ER morphology (Bergmann and Singer 1983). This hypothesis is further supported by the demonstration that overexpression of the SYNV N, P, sc4, or M proteins did not result in obvious effects on plant or yeast cell membranes (Goodin et al. 2001, 2002).

More intriguing than the possible effects of overexpressing individual viral proteins was the different patterns of GFP accumulation within nuclei produced in the context of infection with PYDV or SYNV (Fig. 1). These differences may reflect the manner in which these two viruses undergo morphogenesis. Based on this hypothesis, we propose a model that accounts for the observed differences in patterns of GFP accumulation in virus-infected cells (Fig. 6). Following infection by a nucleorhabdovirus (Fig. 6-1), nuclei enlarge during viroplasm formation and virus replication. Prior to morphogenesis, the $G$ protein must accumulate on the inner nuclear membrane, because this is the only membrane that is crossed by condensed nucleocapsids as they bud into the perinuclear space (MacLeod et al. 1966; Martins et al. 1998; van Beek et al. 1985). Based on our microscopic evidence, we propose that, during morphogenesis of SYNV, the inner nuclear membrane may invaginate into the nucleus; whereas, during maturation of PYDV particles, this may not occur. Instead, accumulation of PYDV virions may result in an expansion of the outer nuclear membrane, as was suggested by 3-D projections of confocal sections (Fig. 2). Both of these models of morphogenesis are consistent with electron microscopy studies that showed that both PYDV and SYNV virions accumulate in the perinuclear space between the inner and outer nuclear membranes. In contrast to nucleorhabdovirusmediated GFP relocalization, infection of plants by viruses that replicate in the cytoplasm induces proliferation of ER membranes via activity of their replicase proteins or $\mathrm{G}$ proteins (Fig. 6-2), as we have shown for TEV-infected plants (Fig. 5Q through S). Consistent with our model was the observation that co-inoculation of 16c plants with TEV and SYNV resulted in an "additive" pattern of GFP localization (Fig. 5T). As previously noted, TEV replication takes place on proliferated ER membranes in the cytoplasm, whereas SYNV induced formation of intranuclear spherules. Both patterns of GFP relocalization were evident in cells of systemically infected leaves, suggesting that replication of both viruses, ER proliferation, and membrane accumulation within nuclei can occur within the same cell, if not simultaneously.

Although this study concentrated primarily on the changes in nuclear membrane morphology in rhabdovirus-infected cells, we have examined all tissues in both SYNV- and PYDVinfected plants in order to provide insight into plant tissue and cell types that become infected by these viruses (Fig. 3). In addition to leaf epidermal cells, GFP accumulated in nuclei of trichome and root cells. By monitoring changes in the patterns of GFP fluorescence, we have no evidence for invasion of root or shoot meristematic tissues by either SYNV or PYDV. However, this may be due to the fact that GFP expression was nearly undetectable in these tissues, presumably due to reduced activity of the $35 \mathrm{~S}$ promoter in these tissues. Further experiments, including immunolocalization, are required to determine whether plant rhabdoviruses enter meristematic tissues.

In conclusion, we hypothesize that GFP in nucleorhabdovirus-infected cells remains associated with virions when the host-derived envelope and glycoprotein are acquired.
This association currently is being investigated. Finally, the dramatic changes in the morphology of rhabdovirus-infected nuclei raises the question of whether or not such nuclei remain transcriptionally active. Although Northern hybridization experiments (data not shown) suggest that GFP transcription is not shut off in SYNV-infected 16c plants, additional experiments are required to connect the spatial and temporal relationships between host gene expression and the cell biology described herein.

\section{MATERIALS AND METHODS}

\section{Plant material, growth conditions, and} virus inoculation procedures.

$N$. benthamiana 16c plants (Ruiz et al. 1998) that constitutively express a GFP variant of the GFP targeted to the ER under the control of the Cauliflower mosaic virus $35 \mathrm{~S}$ promoter (Siemering et al. 1996) were grown in the greenhouse under ambient conditions. These plants were inoculated with virus when they had four to six fully expanded leaves. Virusinfected leaves served as sources of inocula for all viruses, except TRV, which was "agroinoculated" as described by Liu and associates (2002). Plant tissues containing SYNV or INSV were ground in a mortar, with a pestle, containing roughly two volumes of a buffer consisting of $10 \mathrm{mM}$ sodium phosphate, pH $6.9,0.5 \%$ wt/vol $\mathrm{NaSO}_{3}$, and $1 \%$ celite. PYDV-infected $N$. rustica leaves were ground in a mortar, with a pestle, in approximately two volumes of $0.1 \mathrm{M}$ sodium phosphate, $\mathrm{pH} 6.9$. Leaf homogenate was applied to carborundum-dusted leaves of $N$. benthamiana plants from which the apical meristem had been removed prior to inoculation. TEV-infected material was ground in $10 \mathrm{mM}$ sodium phosphate buffer, $\mathrm{pH}$ 6.9. Mock inoculations were conducted using virus-free leaves processed in the same buffers.

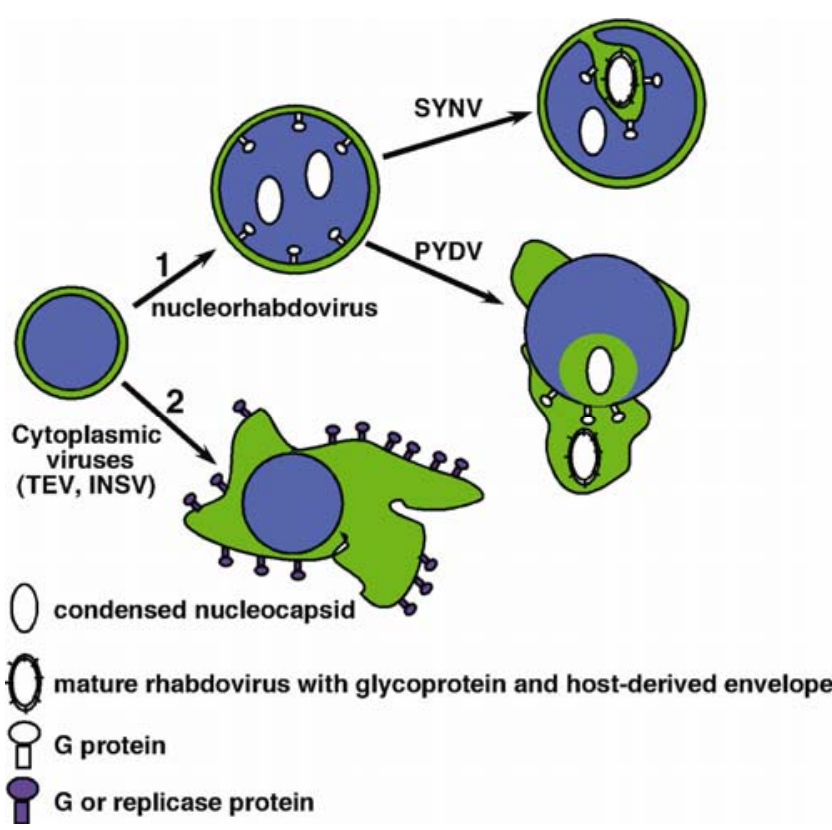

Fig. 6. Model for accumulation of green fluorescent protein (GFP) in nuclei of rhabdovirus-infected cells. GFP associated with endoplasmic reticulum and nuclear membranes is shown in green and the nuclear DNA is shown in blue. Rhabdoviral G proteins are shown in white. Replicase or $\mathrm{G}$ proteins viruses that replicate in the cytoplasm are shown in purple. SYNV = Sonchus yellow net virus, PYDV = Potato yellow dwarf virus, and TEV = Tobacco etch virus INSV = Impatiens necrotic spot virus. 
Loading of cells with fluorescent dyes.

Selective staining of DNA or endomembranes in live cells was accomplished using the cell permeate dyes DAPI or CellTrace BODIPY TR methyl ester (CELL-TRACE), respectively (Molecular Probes, Eugene, OR, U.S.A.). Solutions of CELL-TRACE $(0.25 \mathrm{mM})$ or DAPI $(15 \mu \mathrm{g} / \mathrm{ml})$ were prepared in "agroinfiltration buffer" (10 mM $\mathrm{MgCl}_{2}, 10 \mathrm{mM}$ MES, pH 5.9), and infiltrated into leaves essentially as that described by Goodin and associates (2002). Both dyes were mixed when double labeling was necessary. Following infiltration, the plants were incubated in the dark for 1 to $2 \mathrm{~h}$ before leaf tissue was examined by eplifluoresence microscopy.

\section{Tissue fixation and co-immunolocalization.}

Mock-inoculated or virus-infected leaves were fixed and embedded in paraffin, essentially as described by Perry and associates (1996). Briefly, tissue was cut into small squares (approximately $25 \mathrm{~mm}^{2}$ ) and fixed overnight in freshly prepared $4 \%$ paraformaldehyde dissolved in $50 \mathrm{mM}$ potassium phosphate buffer $(\mathrm{pH} 7.0)$ at $4^{\circ} \mathrm{C}$. Fixed tissue was dehydrated using a series of incubations in ethanol of increasing concentration from 5 to $70 \%$, followed by infiltration with HemoDe (Fisher Scientific, Fairlawn, NJ, U.S.A.). Infiltrated tissue then was incubated in the presence of Paraplast Plus (with dimethyl sulfoxide) dissolved in HemoDe. Following embedding, 10- $\mu \mathrm{m}$ sections were prepared using a Leica Microtome and fixed on ProbeOn Plus microscope slides (Fisher Scientific). Following dewaxing and treatment with Proteinase K, the sections were incubated in the presence of polyclonal antibodies against the SYNV N protein or GFP that were raised in rabbits (immunoglobulin $G$ ) or chickens (immunoglobulin Y), respectively. Goat anti-rabbit secondary antibodies conjugated to QuantumDot 605 (Quantum Dot Corporation, Hayward, CA) and goat anti-chicken secondary antibodies conjugated to AlexaFluor488 (Molecular Probes) were used to develop the immunolocalizations. Prepared slides were mounted in $90 \%$ glycerol containing DAPI at 15 $\mu \mathrm{g} / \mathrm{ml}$ and examined by epifluorescence microscopy.

\section{Epifluorescence microscopy.}

Epifluorescence micrographs were acquired using an Axiocam MR monochromatic digital camera mounted on a motorized Zeiss Axioplan 2 microscope. Both camera and microscope were controlled by Axiovision software (version 4.2). False colors (green for GFP, blue for DAPI, red for CELLTRACE, and gray for differential contrast images) of micrographs also were assigned with Axiovision software. Filter sets that permitted viewing of relevant fluors all were purchased from Chroma (Rockingham, VT, U.S.A.). i) Filter set \#31001 for viewing GFP consisted of a D470/40X excitation (Ex) filter, a 505 DCLP dichroic, and a D540/40X emission (Em) filter. ii) Filter set \#31000 for detecting DAPI fluorescence consisted of a D360/40X Ex filter, a 400 DCLP dichroic, and a D460/50M Em filter. iii) For viewing fluorescence from CELL-TRACE, filter set \#41035, consisting of a HQ546/12X Ex filter, a Q560LP dichroic, and a HQ650/75M Em filter was used. Lenses used in this study included the Plan Neofluar 10X/0.3, Plan Neofluar 25X/0.8 multiple immersion lens, used primarily with water, and Plan Apochromat 100X/1.4 oil immersion lens. Small $\left(1 \mathrm{~cm}^{2}\right)$ pieces of plant tissue were mounted in water on standard glass slides. Leaves were mounted so that the abaxial surface was viewed. Micrographs were exported from the Axiovision software as TIFF files. All subsequent cropping and image manipulations were carried out in Photoshop 7.0 and Canvas 8.0 (Deneba Software, Miami, FL, U.S.A.).

\section{LSCM.}

Confocal microscopy was performed on an Olympus FV1000 (Olympus America Inc., Melville, NY, U.S.A.). DAPI and GFP were excited using 405- and 488-nm laser lines, respectively. Fluorescence emission was captured using filter sets comparable with those described above. Briefly, emission fluorescence longer than $450 \mathrm{~nm}$ was passed through a primary dichroic to photomultipliers 1 and 2 before which secondary dichroics, dispersion gratings, and slits were set to collect 509 $\pm 10 \mathrm{~nm}(\mathrm{GFP})$ and $461 \pm 10 \mathrm{~nm}$ (DAPI) fluorescence, respectively. Lenses used included the Olympus Plan APO 60XWater (NA 0.9), Plan APO 20X-Water (NA 0.75), and UPLAN APO 10X (NA 0.4) objectives.

\section{3-D image reconstruction of confocal images.}

Three-dimensional images were produced from serial optical sectioning of DAPI-stained nuclei using LSCM. Briefly, 29 $0.3-\mu \mathrm{m}$ sections ( $\mathrm{z}$-stack) were captured and exported as TIFF files for both green (GFP) and blue (DAPI). The images then were imported into Image Pro Plus with 3-D plug-in (Media Cybernetics, Silver Spring, MD, U.S.A.). Projections of 3-D reconstructions were exported as TIFF files.

\section{ACKNOWLEDGMENTS}

This manuscript is published with the approval of the Director of the Kentucky Agricultural Experiment Station as Journal Article 04-12-120. This research was supported by grant 5-41184 from the Kentucky Tobacco Research and Development Center awarded to M. Goodin. We would like to thank L. Armogida and M. Frame of BB Microsystems and Olympus USA, respectively, for their excellent technical assistance with confocal imaging; and D. Baulcombe for making available the $N$. benthamiana $16 \mathrm{c}$ plants. We would like to convey our sincere gratitude to A. Jackson (University of California-Berkeley) and H.-t. Hsu (USDA-ARS-FNPRU) for providing us with SYNV and PYDV, respectively. Finally, we wish to thank S. Perry (Department of Agronomy, University of Kentucky) for providing training in the technique of co-immunolocalization; and A. Clark, S. Ghabrial, and D. Smith for critically reviewing the manuscript.

\section{LITERATURE CITED}

Baunoch, D. A., Das, P., Browning, M. E., and Hari, V. 1991. A temporal study of the expression of the capsid, cytoplasmic inclusion and nuclear inclusion proteins of tobacco etch potyvirus in infected plants. J. Gen. Virol. 72:487-492.

Bergmann, J. E., and Singer, S. J. 1983. Immunoelectron microscopic studies of the intracellular transport of the membrane glycoprotein $(\mathrm{G})$ of vesicular stomatitis virus in infected Chinese hamster ovary cells. J. Cell. Biol. 97:1777-1787.

Brigneti, G., Voinnet, O., Li, W. X., Ji, L. H., Ding, S.-W., and Baulcombe, D. C. 1998. Viral pathogenicity determinants are suppressors of transgene silencing in Nicotiana benthamiana. EMBO (Eur. Mol. Biol Organ.) J. 17:6739-6746

Carette, J. E., Stuiver, M., Van Lent, J., Wellink, J., and Van Kammen, A. 2000. Cowpea mosaic virus infection induces a massive proliferation of endoplasmic reticulum but not Golgi membranes and is dependent on de novo membrane synthesis. J. Virol. 74:6556-6563.

Christie, R. G., and Edwardson, J. R. 1977. Light and electron microscopy of plant virus inclusions. Fla. Agric. Exp. Stn. Monogr. Ser. 9:130.

Collings, D. A., Carter, C. N., Rink, J. C., Scott, A. C., Wyatt, S. E., and Allen, N. S. 2000. Plant nuclei can contain extensive grooves and invaginations. Plant Cell. 12:2425-2440.

Dayel, M. J., Hom, E. F., and Verkman, A. S. 1999. Diffusion of green fluorescent protein in the aqueous-phase lumen of endoplasmic reticulum. Biophys. J. 76:2843-2851.

Ellenberg, J, and Lippincott-Schwartz, J. 1999. Dynamics and mobility of nuclear envelope proteins in interphase and mitotic cells revealed by green fluorescent protein chimeras. Methods 19:362-372.

Ellenberg, J., Siggia, E. D., Moreira, J. E., Smith, C. L., Presley, J. F., Worman, H. J., and Lippincott-Schwartz, J. 1997. Nuclear membrane dynamics and reassembly in living cells: targeting of an inner nuclear membrane protein in interphase and mitosis. J. Cell. Biol. 138:1193-1206.

Goldberg, K. B., Modrell, B., Hillman, B. I., Heaton, L. A., Choi, T. J., and Jackson, A. O. 1991. Structure of the glycoprotein gene of Sonchus 
yellow net virus, a plant rhabdovirus. Virology 185:32-38.

Goodin, M. M., Austin, J., Tobias, R., Fujita, M., Morales, C., and Jackson, A. O. 2001. Interactions and nuclear import of the a $\mathrm{N}$ and $\mathrm{P}$ proteins of Sonchus yellow net virus, a plant nucleorhabdovirus. J. Virol. 75:93939406.

Goodin, M. M., Dietzgen, R. G., Schichnes, D., Ruzin, S., and Jackson, A. O. 2002. pGD vectors: versatile tools for the expression of green and red fluorescent protein fusions in agroinfiltrated plant leaves. Plant $\mathbf{J}$. 31:375-383.

Hammond, D. C., Evans, R. K., and Lesnaw, J. A. 1992a. The L protein of vesicular stomatitis virus transcription complexes is specifically photolabeled by 5-azido-uridine 5'-triphosphate, an analogue of the RNA polymerase substrate uridine 5'-triphosphate. J Gen Virol. 73:61-66.

Hammond, D. C., Haley, B. E., and Lesnaw, J. A. 1992b. Identification and characterization of serine/threonine protein kinase activity intrinsic to the L protein of vesicular stomatitis virus New Jersey. J. Gen. Virol. 73:67-75.

Hillman, B. I., Heaton, L. A., Hunter, B. G., Modrell, B., and Jackson, A. O. 1990. Structure of the gene encoding the M1 protein of Sonchus yellow net virus. Virology 179:201-207.

Hsu, H. T., and Black L. M. 1973. Inoculation of vector cell monolayers with potato yellow dwarf virus. Virology 52:187-198.

Hsu, H. T., and Black, L. M. 1974. Multiplication of potato yellow dwarf virus on vector cell monolayers. Virology 59:331-334.

Ismail, I. D., Hamilton, I. A., Robertson, E., and Milner, J. J. 1987. Movement and intracellular location of Sonchus yellow net virus within infected Nicotiana edwardsonii . J. Gen. Virol. 68:2429-2438.

Jackson, A. O., Francki, R. I. B., and Zuidema, D. 1987. Biology, structure and replication of plant rhabdoviruses. Pages 427-508 in: The Rhabdoviruses. R. R. Wagner, ed. Plenum Press, New York.

Li, X. H., Valdez, P., Olvera, R. E., and Carrington, J. C. 1997. Functions of the tobacco etch virus RNA polymerase (NIb): subcellular transport and protein-protein interaction with $\mathrm{VPg} /$ proteinase (NIa). J. Virol. 71:1598-1607.

Liu, Y., Schiff, M., and Dinesh-Kumar, S. P. 2002. Virus-induced gene silencing in tomato. Plant J. 31:777-786.

MacLeod, R., Black, L. M., and Moyer, F. H. 1966. The fine structure and intracellular localization of potato yellow dwarf virus. Virology 29:540552 .

Martins, C. R., Johnson, J. A., Lawrence, D. M., Choi, T. J., Pisi, A. M. Tobin, S. L., Lapidus, D., Wagner, J. D., Ruzin, S., McDonald, K., and Jackson, A. O. 1998. Sonchus yellow net rhabdovirus nuclear viroplasms contain polymerase-associated proteins. J. Virol. 72:5669-5679.

Melcher, U. 2000. The ' $30 \mathrm{~K}$ ' superfamily of viral movement proteins. J. Gen. Virol. 81:257-266.

Perry, S. E., Nichols, K.W., and Fernandez. D. E. 1996. The MADS domain protein AGL15 localizes to the nucleus during early stages of seed development. Plant Cell 8:1977-1989.

Petersen, J. M., Her, L. S., Varvel, V., Lund, E., and Dahlberg, J. E. 2000. The matrix protein of vesicular stomatitis virus inhibits nucleocytoplas- mic transport when it is in the nucleus and associated with nuclear pore complexes. Mol. Cell. Biol. 20:8590-8601.

Petersen, J. M., Her, L. S, and Dahlberg, J. E. 2002. Multiple vesiculoviral matrix proteins inhibit both nuclear export and import. Proc. Natl. Acad. Sci. U.S.A. 98:8590-8595.

Qanungo, K. R., Shaji, D., Mathur, M., and Banerjee, A. K. 2004. Two RNA polymerase complexes from vesicular stomatitis virus-infected cells that carry out transcription and replication of genome RNA. Proc. Natl. Acad. Sci. U.S.A. 101:5952-5957.

Reichel, C., and Beachy, R. N. 1998. Tobacco mosaic virus infection induces severe morphological changes of the endoplasmic reticulum. Proc. Natl. Acad. Sci. U.S.A. 95:11169-11174.

Restrepo-Hartwig, M., and Ahlquist, P. 1996. Brome mosaic virus helicase- and polymerase-like proteins colocalize on the endoplasmic reticulum at sites of viral RNA synthesis. J. Virol. 70:8908-8916.

Restrepo-Hartwig, M., and Ahlquist, P., 1999. Brome mosaic virus RNA replication proteins $1 \mathrm{a}$ and $2 \mathrm{a}$ colocalize and 1a independently localizes on the yeast endoplasmic reticulum. J. Virol. 73:10303-10309

Ruiz, M. T., Voinnet, O., and Baulcombe, D. C. 1998. Initiation and maintenance of virus-induced gene silencing. Plant Cell 10:937-946.

Schaad, M. C., Haldeman-Cahill, R., Cronin, S., and Carrington, J. C. 1996. Analysis of the $\mathrm{VPg}$-proteinase (NIa) encoded by tobacco etch potyvirus: effects of mutations on subcellular transport, proteolytic processing, and genome amplification. J. Virol. 70:7039-7048.

Scholthof, K. B., Hillman, B. I., Modrell, B., Heaton, L. A., and Jackson, A. O. 1994. Characterization and detection of sc4: a sixth gene encoded by Sonchus yellow net virus. Virology 204:279-288.

Schwartz, M., Chen, J., Janda, M., Sullivan, M., den Boon, J., and Ahlquist, P. 2002. A positive-strand RNA virus replication complex parallels form and function of retrovirus capsids. Mol. Cell. 9:505-514.

Schwartz, M., Chen, J., Lee, W.M., Janda, M., and Ahlquist, P. 2004. Alternate, virus-induced membrane rearrangements support positivestrand RNA virus genome replication. Proc. Natl. Acad. Sci. U.S.A. 101:11263-11268.

Siemering, K. R., Golbik, R., Sever, R., and Haseloff, J. 1996. Mutations that suppress the thermosensitivity of green fluorescent protein. Curr. Biol. 6:1653-1663.

Turner, K. A., Sit, T. L., Callaway, A. S., Allen, N. S., and Lommel, S. A 2004. Red clover necrotic mosaic virus replication proteins accumulate at the endoplasmic reticulum. Virology 320:276-290.

van Beek, N. A. M., Lohuis, D., Dijkstra, J., and Peters, D. 1985. Morphogenesis of Sonchus yellow net virus in cowpea protoplasts. J. Ultrastruct. Res. 90:294-303 .

Wagner, J. D. O., Choi, T.-J., and Jackson, A. O. 1996. Extraction of nuclei from sonchus yellow net rhabdovirus-infected plants yields a polymerase that synthesizes viral mRNAs and polyadenylated plus-strand leader RNA. J. Virol. 70:468-477.

Wagner, J. D. O., and Jackson, A. O. 1997. Characterization of the components and activity of sonchus yellow net rhabdovirus polymerase. J. Virol. 71:2371-2382. 